Reprinted with permission from: Physiologia Plantarum. 1997. 101:425-433.

Published and Copyright by: ${ }^{\circledR}$ Physiologia Plantarum. Printed in Denmark - all rights reserved

\title{
2,4-Dichlorophenoxy acetic acid and indoleacetic acid partially counteract inhibition of organogenesis by difluoromethylornithine ${ }^{1}$
}

\author{
DAVID G. DAVIS \\ USDA-ARS Biosciences Research Laboratory, State Univ. Station, Fargo, ND 58105-5674, USA \\ (e-mail:davisd@fargo.ars.usda.gov)
}

\begin{abstract}
:
Difluoromethylornithine (DFMO) is a well known inhibitor of putrescine biosynthesis that has been reported to interact in varying ways with auxins such as indoleacetic acid (IAA) and 2,4-dichlorophenoxy acetic acid (2,4D). In the present report DFMO is shown to inhibit root formation in isolated hypocotyl segments of Euphorbia esula L. (leafy spurge) grown in the dark on solidified nutrient media in Petri dishes. Shoot formation was only slightly inhibited by DFMO and only on media with salts and vitamins diluted 10-fold. 2,4-D inhibited both root and shoot formation in full strength or diluted media. Simultaneous application of both compounds resulted in partial reversal of root inhibition, but only at $450 \mathrm{n} M 2,4-\mathrm{D}$, the highest concentration used. In both media IAA also partially reversed DFMO effects on root formation. The effects of DFMO, 2,4-D or IAA on root (or shoot) formation do not appear to be closely related to endogenous content of the polyamines determined by high performance liquid chromatography.
\end{abstract}

\section{Abbreviations:}

DFMA， DL- $\alpha$-difluoromethylarginine; DFMO, DL- $\alpha$-difluoromethylornithine; PCA, perchloric acid; putrescine, 1,4-butanediamine; spermidine, N-(3-aminopropyl)-1,4-butanediamine; spermine, N,N-bis(3aminopropyl)-1,4-butanediamine.

\footnotetext{
${ }^{1}$ Received 5 November, 1996; revised 22 May, 1997.
} 


\section{Keywords:}

Auxin, 2,4-dichlorophenoxy acetic acid, difluoromethylornithine, Euphorbia esula, leafy spurge, organogenesis, polyamines, putrescine, spermidine, spermine.

\section{Introduction}

Leafy spurge (Euphorbia esula L.) is an economically important perennial weed introduced from Eurasia (Watson 1985). The vigorous spread of leafy spurge is due primarily from buds formed on an extensive root system (Raju 1985). Auxinic herbicides such as 2,4-dichlorophenoxy acetic acid (2,4-D), picloram and dicamba are the compounds most used for control (Lym and Messersmith 1994). All parts of etiolated, aseptically germinated seedlings are capable of regenerating new plants, and hypocotyl segments have been used to determine the effects of auxins and cytokinins on organ formation (Davis and Olson 1993).

The role of polyamines in the formation of roots and shoots also was investigated, with no obvious correlations between endogenous levels of the polyamines and organogenesis (Davis and Olson 1994). However, two inhibitors of putrescine biosynthesis, DL$\alpha$-difluoromethylornithine (DFMO) and DL- $\alpha$-difluoromethylarginine (DFMA) also inhibit leafy spurge root and shoot formation, with the extent of inhibition depending on the concentrations of the two compounds and the organ being observed. DFMO and DFMA are putative specific suicide inhibitors of ornithine decarboxylase (ODC) and arginine decarboxylase (ADC), respectively. ADC and ODC regulate the two major pathways of putrescine biosynthesis in plants (Slocum 1991). A DFMA-induced inhibition of root formation was reversed by the addition of putrescine, whereas the DFMO-induced inhibition of root formation was not (Davis and Olson 1994).

The effects of DFMO on different plant systems are extremely variable, ranging from inhibition to no effect to stimulatory, and are a function of concentration and the particular plant system being tested. For example, DFMO $(1 \mathrm{~m} M)$ had no effect on development and growth of grapevine microcuttings in vitro, as opposed to DFMA, which inhibited development (Martin-Tanguy and Carre 1993). In carrot (Daucus carota L.), $5 \mathrm{mM}$ DFMO inhibited ADC and ODC in vitro but not in vivo (Mengoli et al. 1989), and $5 \mathrm{mM}$ DFMO enhanced somatic embryogenesis in carrot cell suspension cultures (Nissen and Minocha 1993). Root growth was inhibited by DFMO in germinating seeds of Arabidopsis thaliana (Mirza and Bagni 1991). In calli of the rubber tree (Hevea brasiliensis), a member of the Euphorbiacea as is leafy spurge, DFMO treatment actually increased putrescine content, but inhibited somatic embryogenesis (EI Hadrami and Anzac 1992).

Indole-3-acetic acid (IAA) partially counteracts the inhibition of organogenesis in leafy spurge tissues resulting from treatment with $0.5 \mathrm{~m} M$ DFMO $+0.5 \mathrm{~m} M$ DFMA in the culture medium (Davis and Olson 1994). This treatment resulted in the depletion of endogenous putrescine within approximately 3 days.

Page 2 of 13 
2,4-D has some auxin-like properties at low concentrations and is herbicidal at high concentrations. In many plant systems 2,4-D is often the auxin of choice to induce callus and maintain cell suspension cultures of many plant species, including carrot (Nissen and Minocha 1993) and leafy spurge (Davis et al. 1988, Evenson et al. 1988). 2,4-D can replace the need for exogenous auxins and cytokinins in the growth and maintenance of leafy spurge cell suspension cultures (Davis et al. 1988, Evenson et al. 1988). In leafy spurge hypocotyl segments, $230 \mathrm{n} M$ 2,4-D inhibited root and shoot formation with no stimulation of root formation at any concentration (Davis and Olson 1993). In this way 2,4-D affected leafy spurge hypocotyl segments differently than IAA, which consistently stimulated root formation with or without DFMO+DFMA present (Davis and Olson 1994), although at a reduced level in the presence of both inhibitors. In micropropagated poplar shoots in vitro, complex interactions were observed between auxin (naphthaleneacetic acid) and inhibitors of polyamine biosynthesis (Hausman et al. 1995a,b).

Because both 2,4-D and DFMO inhibited organogenesis in leafy spurge isolated hypocotyl segments, the report by Nissen and Minocha (1993) that inhibition of somatic embryogenesis in carrot cell suspension cultures by 2,4-D could be reversed by DFMO was of interest. The present research was done to establish the relationship between DFMO and 2,4-D in leafy spurge. The results with 2,4-D were also compared to those of IAA, although in separate experiments and at different concentrations, due to the different sensitivities of the tissues to the two compounds.

\section{Materials and methods}

Seeds collected from a field plot in southeastern North Dakota were surface sterilized with $70 \%$ ethanol for 1 minute followed by 20 minutes in 3.15\% Na hypochlorite and three rinses in sterile water. The high concentration of bleach was required to eliminate a seed-borne contaminant. The seeds were germinated on sterile agar-water $(0.7 \%, \mathrm{w} / \mathrm{v})$ in darkness at $30 / 20^{\circ} \mathrm{C}(12 / 12$ hours). After 2 weeks, the etiolated hypocotyls were cut into $1-\mathrm{cm}$ lengths and placed onto $0.7 \%$ agar medium with B5 vitamins and salts, with $2 \%$ (w/v) sucrose (Gamborg et al. 1968). Filter-sterilized aqueous solutions of DFMO and acetone solutions of IAA were dispersed into autoclaved medium while it was still liquid. The sodium salt of 2,4-D was added to $2 \mathrm{x}$ strength $\mathrm{B} 5$ medium, autoclaved, and added to equal amounts of $4 \%$ sucrose that was autoclaved separately, resulting in $1 \times(100 \%)$ strength B5 medium with $2 \%$ sucrose.

Two levels of nutrition were used in these experiments: 100\% B5 vitamins and minerals, and 10\% B5 vitamins and minerals, a level of nutrition that was marginal for organogenesis. Sucrose remained at $2 \%(\mathrm{w} / \mathrm{v})$ throughout. The hypocotyl segments were grown in plastic Petri dishes $\left(6 \mathrm{~cm}\right.$ diameter) which were wrapped with Parafilm ${ }^{\circledR}$ and aluminum foil, and incubated in darkness at $26^{\circ} \mathrm{C}$. Roots and shoots were observed at 7 and (usually) 28 days by the use of a dissecting microscope and they were counted when they were clearly recognizable as distinct organs $(\geq 1-2 \mathrm{~mm}$ long).

Nutrients for the B5 medium were plant tissue culture tested by Sigma Chemical Co. Polyamines were obtained from Sigma, or Aldrich Chemical Co., Inc. (Milwaukee, WI, USA). DFMO was obtained from Marion-Merrill-Dow (Cincinnati, OH, U.S.A.).

Page 3 of 13 
Experiments were run two or more times. Eight hypocotyl segments were used per Petri dish, with 10 dishes per treatment. Triplicate samples ( 3 dishes) were used to determine polyamine concentrations. Comparisons were made to the controls or between treatments using two-sample $t$-tests with the computer program Statistix (NH Analytical Software, St. Paul, MN, U.S.A.).

Endogenous polyamines were quantified as dansylated derivatives using high performance liquid chromatography (HPLC). Hypocotyl segments were weighed, frozen at $-20^{\circ} \mathrm{C}$ and analyzed as described by Minocha et al. (1990). Later samples were stored at $-80^{\circ} \mathrm{C}$ prior to polyamine analysis. The freeze-thaw method has been shown to be as good or superior to homogenization for extraction of polyamines in tissues from 10 different plant tissues (Minocha et al. 1994). Briefly, analysis was done on samples thawed and frozen 3 times in 5\% perchloric acid (PCA). Similar results were obtained with samples freeze-thawed 3 times and those ground for 2 minutes in a glass homogenizer, so the freeze-thaw method was used for all samples. Conjugated polyamines from PCA-soluble and PCA-insoluble extracts were determined from samples hydrolyzed 24 hours in $6 \mathrm{M}$ $\mathrm{HCl}$ at $110^{\circ} \mathrm{C}$, and dansylated for HPLC, as above.

The HPLC system consisted of a Perkin-Elmer Series 400 pump, fitted with a 10- $\mu 1$ injection loop, a $\mathrm{C}_{18}$ reversed-phase column (Perkin-Elmer CRC 18, particle size $3 \mu \mathrm{m}$ ), and a Perkin-Elmer fluorescence detector (excitation at $340 \mathrm{~nm}$, emission at $515 \mathrm{~nm}$ ). The polyamines were eluted using a linear gradient of acetonitrile and heptanesulfonate (10 mM in water, $\mathrm{pH} 3.4$ ) at a flow rate of $2.5 \mathrm{ml} \mathrm{minutes}^{-1}$. Heptanediamine was used as an internal standard for polyamine analysis, with the elution times for putrescine, heptanediamine, spermidine and spermine being 2.24, 2.71, 3.22 and 4.05 minutes, respectively. Quantification was calculated by comparison to the area of the heptanediamine peak.

\section{Results}

\section{Root formation}

IAA at $0.23 \mu \mathrm{M}$ or greater concentration consistently increased root formation on either 100\% B5 medium (Fig. 1A) or on B5 medium with the salts and minerals diluted 10fold (10\%) (Fig. 1B). This was true in the presence or absence of DFMO, which by itself greatly inhibited root formation (Fig. 1A,B).

On the other hand, 2,4-D decreased root formation with increasing concentration in the absence of DFMO. Greater inhibition of root formation was observed on the $100 \%$ B5 medium (Fig. 1C) than on the 10\% B5 medium (Fig. 1D). DFMO alone caused a 5- to 6-fold inhibition of root formation. Both compounds applied simultaneously (DFMO+2,4-D; with the latter at 0.45 to $45 \mathrm{n} M$, respectively) gave similar results to DFMO on either $10 \%$ or $100 \%$ B5 medium. 

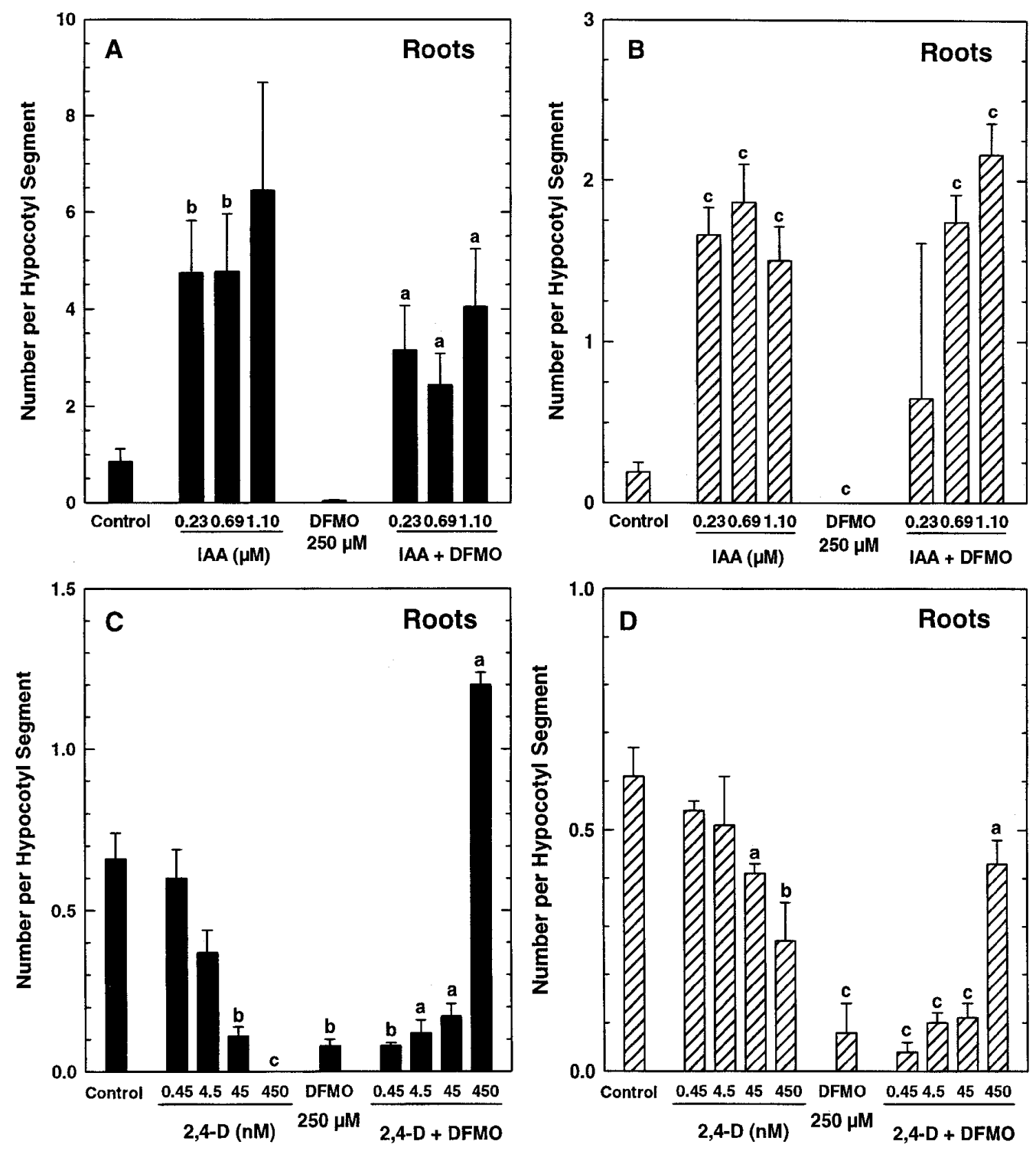

Fig. 1. Effects of (A,B) IAA, DFMO and DFMO+IAA or (C,D) 2,4-D, DFMO and DFMO + 2,4-D on root formation in leafy spurge hypocotyl segments. A and C, 100\% B5 medium. B and $\mathrm{D}, 10 \% \mathrm{~B} 5$ medium. 2,4-D concentrations were $\mathrm{n} M$; all others were $\mu M$. Vertical lines are \pm SE. a, b and c designate significant difference from controls at $P<0.05,0.01$ or 0.001 levels, respectively.

However, at the critical concentration of $450 \mathrm{n} M 2,4-\mathrm{D}$, root formation was greater in the presence of both 2,4-D and DFMO than of DFMO alone. The number of roots formed in the presence of $450 \mathrm{n} M 2,4-\mathrm{D}$ with DFMO present exceeded that of the controls in the 100\% B5 medium (Fig. 1C), but not in the 10\% B5 medium (Fig. 1D). In all treatments the numbers of roots per hypocotyl segment were much less for 2,4-D+DFMO (Fig. 1C,D) than for IAA+DFMO (Fig. 1A,B). 

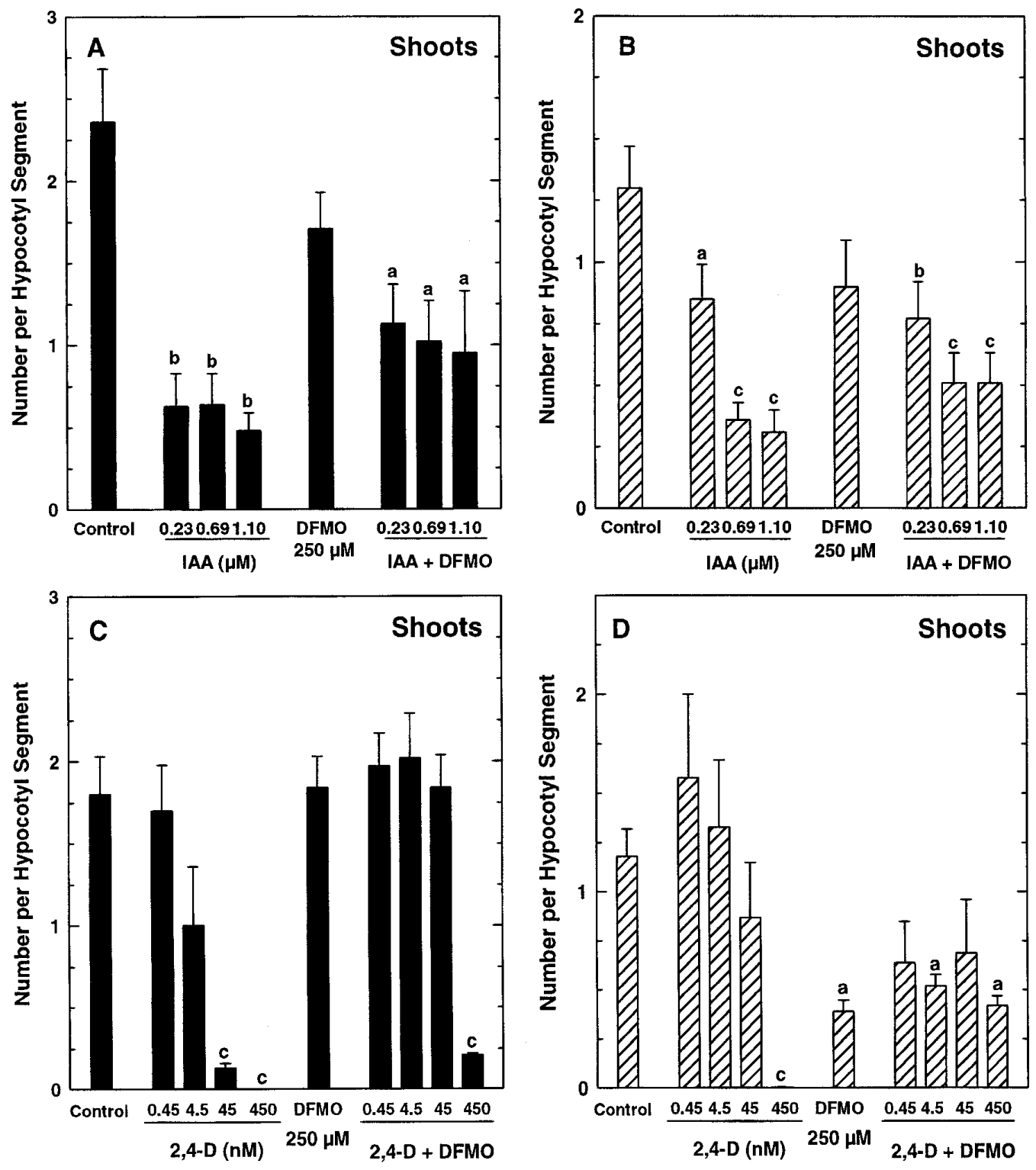

Fig. 2. Effects of (A,B) IAA, DFMO and DFMO+IAA or (C,D) 2,4-D, DFMO and DFMO + 2,4-D on shoot formation in leafy spurge hypocotyl segments. A and C, 100\% B5 medium. B and $\mathrm{D}, 10 \% \mathrm{B5}$ medium. 2,4-D concentrations were $\mathrm{n} M$; all others were $\mu M$. Vertical lines are \pm SE. a, $b$ and c designate significant difference from controls at $P<0.05,0.01$ or 0.001 levels, respectively.

Duplicate experiments were run with either 250 or $500 \mu M$ DFMO. The data, with one exception, are reported only from the experiments with $250 \mu M$ DFMO. The results with the two concentrations of DFMO were similar except that $500 \mu M$ DFMO was generally more inhibitory to shoot formation than $250 \mu M$ DFMO, and shoot formation on $10 \%$ B5 medium was almost totally eliminated by the combination of IAA $+500 \mu M$ DFMO. Also, with IAA $+250 \mu M$ DFMO some shoot formation occurred. 


\section{Shoot formation}

Control hypocotyl segments formed about twice the number of shoots (Fig. 2), as roots (Fig. 1). IAA inhibited shoot formation (Fig. 2A,B).

On 100\% B5 medium DFMO had little or no effect on shoot formation (Fig. 2A,C). In contrast, on 10\% B5 medium DFMO effects varied from a statistically insignificant reduction (Fig. 2B) to more than 50\% inhibition (Fig. 2D). The combination of IAA+DFMO resulted in somewhat fewer shoots than either controls or DFMO alone in the 100\% B5 medium (Fig. 2A). The results with IAA+DFMO were intermediate between those of IAA alone or DFMO alone on both media.

2,4-D at higher concentrations inhibited shoot formation on either $100 \%$ (Fig. 2C) or $10 \%$ (Fig. 2D) B5 media, with no shoots formed in the presence of $450 \mathrm{n} M 2,4-\mathrm{D}$. Unlike the situation for root formation, in 100\% B5 medium 0.45 to $45 \mathrm{n} M 2,4-\mathrm{D}$ with DFMO resulted in shoot numbers approximately equal to that of the control (Fig. 2C). In both media (Fig. 2C,D), $450 \mathrm{n} M$ 2,4-D by itself almost totally inhibited shoot formation. A slight, but insignificant, reversal of the inhibition by $2,4-\mathrm{D}$ was observed on $100 \%$ B5 medium when $450 \mathrm{n} M 2$ 2,4-D was combined with DFMO. On 10\% B5 medium the reversal was more prominent (Fig. 2D), with shoot numbers approximately equal to those obtained with DFMO by itself and still significantly less than the control.

Both IAA and 2,4-D caused callus formation, which was not quantified. The amount of callus appeared to vary with the concentration of IAA or 2,4-D, and in general 2,4-D was far more prone to initiate callus than IAA. DFMO caused little callus formation, mostly on the cut ends of the hypocotyl segments.

\section{Effects of IAA on polyamine levels}

Polyamine concentrations in hypocotyl segments treated for $0,2,5$ and 8 days with DFMO, $1.1 \mu M$ IAA or IAA+DFMO are shown in Fig. 3. Free polyamines, PCA-soluble conjugates and PCA-insoluble conjugates of putrescine and spermidine were obtained. Greater levels of putrescine (free + conjugated) than spermidine were determined at most harvest dates. Spermine was not detected at day 0 , and only free spermine was detected at 2,5 and 8 days of culture.

Putrescine levels in controls and DFMO-treated tissues were similar and declined with time (Fig. 3). In contrast, total putrescine levels increased with time in all tissues treated with only IAA. At day 8 the total putrescine level in IAA-treated tissues was more than twice that in the controls. The free putrescine in the IAA-treated tissues was about three times that in the controls. Total putrescine levels in tissues treated with IAA+DFMO were similar to those of IAA-treated tissues up to 5 days. By day 8 the level of free putrescine was greater in the IAA-treated tissues than in tissues treated with IAA+DFMO. Also at day 8 the insoluble putrescine conjugates in the IAA+DFMOtreated tissues were slightly (but not significantly) greater than in any of the other treatments. 
Spermidine levels in controls and treated tissues increased with time (Fig. 3 ) and in that way differed from putrescine levels. As with putrescine, DFMO had no significant effect on spermidine levels. IAA by itself increased total and free spermidine (Fig. 3). The combination of IAA+DFMO resulted in only a relatively small increase in total spermidine above the controls, with little difference in free spermidine. The total spermidine concentrations in IAA+DFMO-treated tissues were intermediate between those treated with IAA or DFMO alone.

Spermine was not detected at day 0 , and only free spermine was found at 2 , 5 and 8 days of culture, increasing to less than $25 \mathrm{nmol}$ per $\mathrm{g}$ fresh weight by day 8 (Fig. 3). All three treatments (IAA, DFMO and IAA+DFMO) resulted in greater levels of free spernine than the controls, but the differences were not significant between the treatments.

\section{Effects of 2,4-D on free polyamine levels}

Because 2,4-D at 45 and $450 \mathrm{n} M$ and DFMO inhibited root formation on $100 \% \mathrm{~B} 5$ medium, and the combination of $450 \mu M 2,4-\mathrm{D}+\mathrm{DFMO}$ reversed that inhibition, it was of interest to determine if a relationship existed between free polyamines and the effects of the two inhibitors. Only free polyamines were determined for experiments on the effects of 2,4-D and DFMO (Fig. 4) because the changes in free polyamine levels approximated the levels of total polyamines in the IAADFMO study (Fig. 3).

Free putrescine levels in control hypocotyl segments declined with time, but $450 \mathrm{n} M$ 2,4-D increased free putrescine 5- to 6-fold above the controls at 5 to 8 days of culture (Fig. 4). At $45 \mathrm{n} M, 2,4-\mathrm{D}$ had little effect on putrescine levels. The rest of the treatments differed slightly from the controls; DFMO + $450 \mathrm{n} M$ 2,4-D increased free putrescine lev- 
els only slightly. By 14 days the free putrescine levels in tissues treated with $450 \mathrm{n} M$ 2,4-D were reduced to approximately twice that of the controls.

By itself, $450 \mathrm{n} M$ 2,4-D increased the level of free spermidine above the controls at all harvest dates. Spermidine levels were nearly 5 -fold greater than in the controls by day 8 and remained approximately 2-fold greater than controls at day 14 (Fig. 4). Free spermidine was also increased above the controls by DFMO + 2,4-D at $450 \mathrm{n} M$, but to a lesser extent than with $450 \mathrm{n} M 2,4-\mathrm{D}$ alone. The maximum increase occurred at 5 days and was approximately twice the level of the controls. All other treatments (DFMO alone, $45 \mathrm{n} M$ 2,4-D and DFMO + 2,4-D at $45 \mathrm{n} M$ ) had little effect on spermidine levels. As with free putrescine, free spermidine decreased by day 14 in the hypocotyl segments treated with $450 \mathrm{n} M 2,4-\mathrm{D}$.

As with free putrescine and free spermidine, $450 \mathrm{n} M$ 2,4-D also increased free spermine above control levels (Fig. 4). However, free spermine in the $450 \mathrm{n} M$ 2,4-D-treated tissues continued to increase slightly up to 14 days of culture, unlike free putrescine or free spermidine. DFMO + 2,4-D at $450 \mathrm{n} M$ also increased free spermine above control levels, but not to the level of $450 \mathrm{n} M 2,4-\mathrm{D}$.

\section{Discussion}

DFMO is considered as a specific suicide inhibitor of ornithine decarboxylase activity, thereby interfering with putrescine biosynthesis, which affects spermidine, spermine and ethylene biosynthesis (Slocum 1991). Because putrescine can also be biosynthesized in plants via an alternate arginine pathway, treatment of plant tissues with DFMO alone does not guarantee a reduction in putrescine biosynthesis. In leafy spurge hypocotyl segments, $250 \mu M$ DFMO had little or no effect on endogenous levels of putrescine; $500 \mu \mathrm{M}$ DFMO reduced putrescine levels, but not to an undetectable level. In a previous study the combination of DFMO and DFMA (a specific suicide inhibitor of arginine decarboxylase activity) applied together decreased putrescine levels to zero within 5 days (Davis and Olson 1994). DFMO might affect enzymes other than ODC because at a concentration of DFMO that had little or no effect on endogenous putrescine levels DFMO strongly inhibited root formation in the leafy spurge hypocotyl segments. DFMO also inhibited shoot formation in leafy spurge hypocotyl segments grown under conditions of low nutrition.

The impact of DFMO on plant tissues is difficult to assess because it affects different plant tissues in a variety of ways. For example, DFMO inhibited putrescine levels in Euonymus europaeus explants and actually stimulated rooting activity (Bonneau et al. 1995). The interactions between DFMO and the auxins are even more complex. Rather than inhibit somatic embryos of carrot, DFMO increased their vigor (Nissen and Minocha 1993). These same workers showed that the 2,4-D-induced inhibition of carrot somatic embryogenesis can be counteracted by DFMO. Also, the actions of several polar auxin transport inhibitors were counteracted by DFMO in several plant species and developing tissues (Nissen 1996). The conclusions were that 2,4-D probably inhibits plant development by interfering with the internal IAA gradient, and that DFMO may interfere with the action of auxins and phytotropins in some unknown fashion. DFMO inhibited the

Page 9 of 13 
growth of rice cell suspensions only if 2,4-D was absent, with no effect on plant regeneration from callus (Koetje $e t$ al. 1993).

A possible, but untested scenario is that DFMO may inhibit the biosynthesis of IAA, resulting in reduced root formation. Exogenous IAA may have overcome part of the DFMO-induced inhibition of root formation in leafy spurge hypocotyl segments by simply replacing some of the endogenous IAA that would have been biosynthesized in the absence of DFMO. Although exogenous IAA (in the absence of DFMO) induced relatively large numbers of roots compared to controls, it may not have been available in sufficient quantities long enough to completely overcome the inhibitory action of $250 \mu M$ DFMO which was approximately 230 times the concentration of IAA.

The interaction between 2,4-D and DFMO is more difficult to rationalize, because both compounds by themselves inhibited root and shoot formation, with no indication of stimulation by either compound alone at any of the concentra-

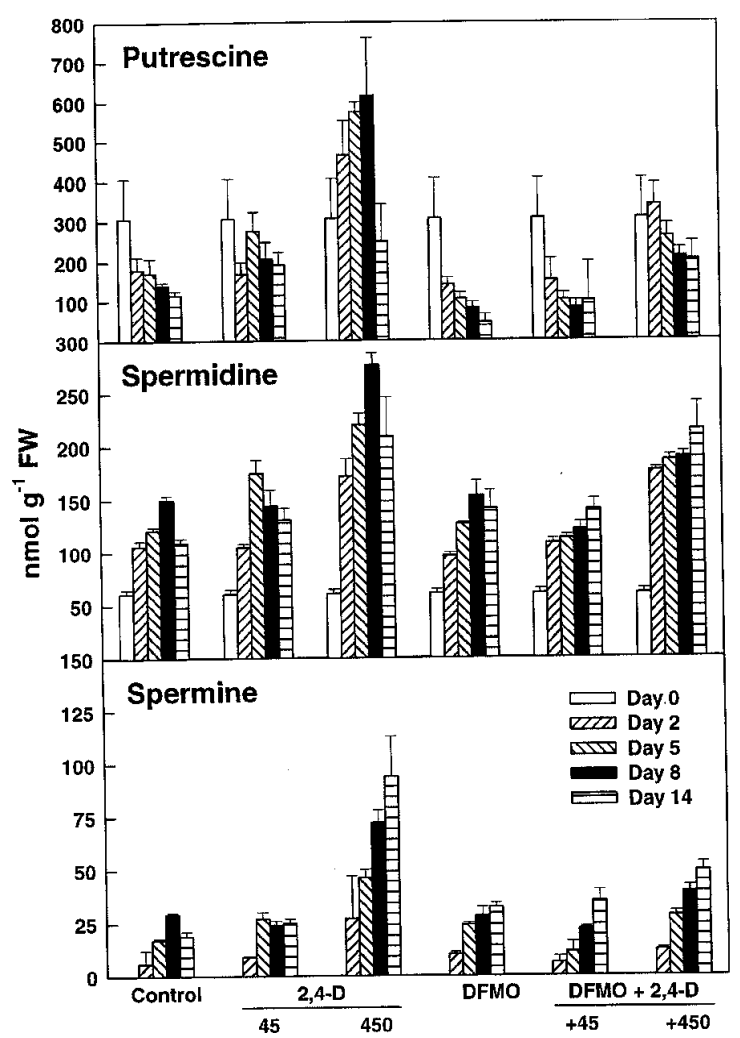

Fig. 4. Free polyamine levels in hypocotyl segments up to 14 days of treatment with DFMO (at $500 \mu M$ ), 2,4-D (at 45 or 450 nM) or 2,4-D + DFMO. Vertical lines are \pm SE. tions used in the present report. 2,4-D inhibits synthesis of DNA, RNA and protein in the bacterium Azospirillum brasilense, and the polyamines prevent the toxicity of 2,4-D in those cells (Castro et al. 1996). There has not been a comparable study utilizing DFMO and 2,4-D. Perhaps DFMO and 2,4-D also interact with each other, but in a different way, preventing full expression of their individual modes of action at critical concentrations. Even at the high concentration of exogenous DFMO, the amount of DFMO translocated to the active site(s) may be quite small. Although 2,4-D and DFMO are applied exogenously at very different concentrations, their internal concentrations at the active site(s) may be similar due to differential absorption, translocation, solubility, individual affinities or levels of activity.

Leafy spurge hypocotyl segments formed roots and shoots over at least a 10-fold range of nutritional levels, although usually fewer roots and shoots were obtained on controls grown on the $10 \%$ B5 medium. The major differences in response to treatment between the two media were that 2,4-D was less inhibitory towards root formation on the $10 \%$ B5 medium than on the 100\% B5 medium, and shoot formation was almost totally eliminated on the $10 \%$ B5 medium when treated with $500 \mu M$ DFMO + IAA. Presumably the concentration of one or more nutrients in the diluted medium is at a threshold level, 
but is sufficient for survival of this perennial weed. It is unclear why 2,4-D would be more inhibitory under limited nutritional levels.

The polyamines have been implicated in plant growth and development, even to the possibility of considering them to be classed as growth regulators or second messengers rather than as plant hormones, which are usually present at much smaller concentrations (Davies 1995). In their 1989 review, Evans and Malmberg indicated that insufficient evidence was accumulated to classify the polyamines as growth regulators.

No close relationship appears to exist between putrescine concentration and organogenesis in leafy spurge hypocotyl segments (Davis and Olson 1994). In control tissues (in the present study) the trend was for putrescine to decline during the first 8 days when both roots and shoots were initiated. The greatest increases in putrescine were in tissues treated with $450 \mathrm{n} M$ 2,4-D,which strongly inhibited both root and shoot formation. Perhaps the increases in putrescine levels were due to stress induced by 2,4-D, an auxinic herbicide. Stresses of various kinds have been shown to increase putrescine levels in plant tissues, such as high $\mathrm{NH}_{4} / \mathrm{NO} \frac{-}{3}$ ratios (Altman and Levin 1993) or endogenous abscisic acid (Aurisano et al. 1993) or a variety of causes, including deficiencies in several mineral elements (various references in Evans and Malmberg 1989 and Flores 1991).

Interactions between putrescine, peroxidase and auxin have been implicated in the regulation of rooting of poplar shoots in vitro (Gaspar et al. 1994, Hausman et al. 1995a,b); putrescine stimulated peroxidase activity, but spermidine did not. An inductive phase of only a few hours on auxin-containing media produced a peak of peroxidase activity prior to rooting (Gaspar et al. 1994, Hausman et al. 1995a,b). The contention is that it is the process of catabolism of putrescine to pyrroline that is involved in induction of rooting, rather than putrescine itself or spermidine or spermine.

Both spermidine and spermine increased in untreated leafy spurge hypocotyl segments during the first 8 days, implying the possibility of direct relationships between shoot formation and the concentration of either spermidine or spermine. If true, spermine could be the most important polyamine involved in organogenesis, although it was present in much lower concentrations than either putrescine or spermidine. Spermine was undetectable in leafy spurge hypocotyl segments prior to initiation of organs but the levels increased up to at least 14 days. This is the most active period of organogenesis in the isolated leafy spurge hypocotyl segments (Davis and Olson 1993 and the present study). However, as with putrescine, the greatest increases of both spermidine and spermine were obtained in tissues treated with $450 \mathrm{n} M$ 2,4-D, which strongly inhibited both roots and shoots. Therefore, the concentrations of neither spermidine nor spermine appear to be primary factors in the formation of shoots. Spermine has been shown to interact with and affect the conformation of DNA (Zhurkin et al. 1980), and the polyamines seem to be required for cell division, growth and development in plants. Recent evidence is that spermine regulates post translational ADC synthesis and therefore may be involved in its anti-senescent properties in Avena sativa L. (cv. Victory) leaves (Borrell et al. 1996). However, the exact roles of spermine and the other polyamines in the development of roots and shoots remain unclear.

Likewise, the mode of action of DFMO and its interaction with the auxins in plants remain unclear. In vertebrates, polyamines appear to act as ion-channel gates to the 
movement and distribution of such ions as $\mathrm{Ca}^{2+}$ (Forsythe 1995). A similar action may also occur in plants, although polyamine action(s) may be multiple and part of their action(s) in plants may be very different from their action(s) in animals. Likewise, the mechanism of DFMO action in plants may also differ from that in animals.

\section{Acknowledgments}

DFMO was kindly provided by Marion-Merrill-Dow. Polyamine analyses for Fig. 3 were performed in the laboratory of Dr. Subhash Minocha under a contract, and for Fig. 4 in the Biosciences Research Laboratory using Dr. Minocha's methods. Dr. Kristi Biewer, Prudence Olson and Cheryl Kimberlin gave excellent technical assistance.

\section{References}

Altman, A. and N. Levin. 1993. Interactions of polyamines and nitrogen nutrition in plants. Physiol. Plant. 89:653-658.

Aurisano, N., A. Bertani, M. Mattana, and R. Reggiani. 1993. Abscisic acid induced stress-like polyamine pattern in wheat seedlings, and its reversal by potassium ions. Physiol. Plant. 89:687-692.

Bonneau, L., N. Beranger-Novat, J. Monin, and J. Martin-Tanguy. 1995. Stimulation of root and somatic embryo production in Euonymus europaeus L. by an inhibitor of polyamine biosynthesis. Plant Growth Regul. 16:5-10.

Borrell, A., R. T. Besford, T. Altabella, C. Masgrau, and A. F. Tiburcio. 1996. Regulation of arginine decarboxylase by spermine in osmotically-stressed oat leaves. Physiol. Plant. 98:105-110.

Castro, S., A. Fabra, G. Mori, V. Rivarola, W. Giordano, and H. Balegno. 1996. Characterization of 2,4-dichlorophenoxyacetic acid transport and its relationship with polyamines in Azospirillum brasilense. Toxicol. Lett. 84:33-36.

Davis, D. G. and P. A. Olson. 1993. Organogenesis in leafy spurge (Euphorbia esula L.). In Vitro Cell. Dev. Biol. 29P: 97-101.

Davis, D. G. and P. A. Olson. 1994. Effects of putrescine and inhibitors of putrescine biosynthesis on organogenesis in Euphorbia esula L. In Vitro Cell. Dev. Biol. 30P:124-130.

Davis, D. G., P. A. Olson, and R. L. Stolzenberg. 1988. Organogenesis in cell cultures of leafy spurge (Euphorbiaceae) accessions from Europe and North America. Plant Cell Rep. 7:253-256.

Davies, P. J. 1995. Plant hormones. In: Plant Hormones: Physiology, Biochemistry and Molecular Biology (P. J. Davies, ed.), 2nd Ed., p. 10. Kluwer Academic Publishers, Dordrecht. ISBN 0-7923-2985-6.

El Hadrami, I. and J. Anzac. 1992. Effects of polyamine biosynthetic inhibitors on somatic embryogenesis and cellular polyamines in Hevea brasiliensis. J. Plant Physiol. 140:33-36.

Evans, P. T. and R. L. Malmberg. 1989. Do polyamines have roles in plant development? Annu. Rev. Plant Physiol. Plant Mol. Biol. 40:235-269.

Evenson, K. J., D. S. Galitz, and D. G. Davis. 1988. The relationship of nitrogen source and in vivo nitrate reductase activity to root formation in Euphorbia esula cell suspension cultures. Plant Cell Rep. 7:361364.

Flores, H. E. 1991. Changes in polyamine metabolism in response to abiotic stress. In: Biochemistry and Physiology of Polyamines in Plants (R. D. Slocum and H. E. Flores, eds), pp. 213-228. CRC Press, Boca Raton, FL. ISBN 0-8493-6865-0. 
Forsythe, I. D. 1995. A physiological function for polyamines? Curr. Biol. 5:1248-1251.

Gamborg, O. L., R. A. Miller, and K. Ojima. 1968. Nutrient requirements of suspension cultures of soybean root cells. Exp. Cell Res. 50:148-151.

Gaspar, T., Kevers, C., Hausman, J. E and Ripetti, V R. 1994. Peroxidase activity and endogenous free auxin during adventitious root formation. In Physiology, Growth and Development of Plants in Culture (R J. Lumsden, J. R. Micholas and W. J. Davies, eds), pp. 289-298. Kluwer Academic Publishers, Dordrecht. ISBN 0-7923-2516-8.

Hausman, J. F., C. Kevers, and T. Gaspar. 1995a. Auxin-polyamine interaction in the control of the rooting inductive phase of poplar shoots in vitro. Plant Sci. 110:63-71.

Hausman, J. F., C. Kevers, and T. Gaspar. 1995b. Putrescine control of peroxidase activity in the inductive phase of rooting in poplar shoots in vitro, and the adversary effect of spermidine. J. Plant Physiol. 146:681-685.

Koetje, D. S., H. Kononowicz, and T. K. Hodges. 1993. Polyamine metabolism associated with growth and embryogenic potential of rice. J. Plant Physiol. 141:215-221.

Lym, R. and C. Messersmith. 1994. Leafy spurge (Euphorbia esula) control, forage production, and economic return with fall-applied herbicides. Weed Technol. 8:824-829.

Martin-Tanguy, J. and M. Carre. 1993. Polyamines in grapevine microcuttings cultivated in vitro. Effects of amines and inhibitors of polyamine biosynthesis on polyamine levels and microcutting growth and development. Plant Growth Regul. 13:269-280.

Mengoli, M., N. Bagni, G. Luccarim, V. Nutti-Ronchi, and D. Serafini-Fracassini. 1989. Daucus carota cell cultures: Polyamines and effect of polyamine biosynthesis inhibitors in the preembryogenic phase and different embryo stages. J. Plant Physiol. 134:389-394.

Minocha, R., W. C. Shortle, S. L. Long, and S. C. Minocha. 1994. A rapid and reliable procedure for extraction of cellular polyamines and inorganic ions from plant tissues. J. Plant Growth Regul. 13:187193.

Minocha, S. C., R. Minocha, and C. A. Robie. 1990. High performance liquid chromotographic method for the determination of dansyl-polyamines. J. Chromatogr. 511:177-183.

Mirza, J. I. and N. Bagni. 1991. Effects of exogenous polyamines and difluoromethylornithine on seed germination and root growth of Arabidopsis thaliana. Plant Growth Regul. 10:163-168.

Nissen, P. 1996. Difluoromethylornithine counteracts effects of auxins and inhibitors of polar auxin transport on plant development. Biol. Plant. 38:343-349.

Nissen, P. and S. C. Minocha. 1993. Inhibition by 2,4-D of somatic embryogenesis in carrot as explored by its reversal by difluoromethylornithine. Physiol. Plant. 89:673-680.

Raju, M. V. S. 1985. Morphology and anatomy of leafy spurge. In: Leafy Spurge, Monograph No. 3 (A. K. Watson, ed.). Weed Science Society of America, Champaign, IL. ISBN 0-911733-03-5.

Slocum, R. D. 1991. Polyamine metabolism in plants. In: Biochemistry and Physiology of Polyamines in Plants (R. D. Slocum and H. E. Flores, eds), pp. 23-40. CRC Press, Boca Raton, FL. ISBN 0-84936865-0.

Watson, A. K. (ed.). 1985. Leafy Spurge, Monograph No. 3. Weed Science Society of America, Champaign, IL. 104 pp. ISBN 0-911733-03-5.

Zhurkin, V. B., Y. P. Lysov, and V. I. Ivanov. 1980. Interaction of spermine with different forms of DNA. A conformational study. Biopolymers 19:1415-1434. 